\title{
Analysis on Existence and Transmission of Tacit Knowledge in Sports Teaching
}

\author{
Lin Ma \\ Taishan University, Taishan 271000, China
}

\begin{abstract}
Tacit knowledge is a kind of knowledge that cannot be explained in words. Sports is a course with sports techniques as the teaching center. As the explicit knowledge has obvious externalization characteristics and is expressed by passing on and learning the sports techniques and the sports activities have strong operability, the sports teaching features are emphasized. With the continuous perfection of sports teaching, "tacit knowledge", as an important part of knowledge system, is more and more valued by the sports teacher. In this paper, the tacit knowledge in the sports teaching is analyzed and the role of tacit knowledge in the information transmission is further studied.
\end{abstract}

Key words: Sports teaching; tacit knowledge; existence; transmission

Tacit knowledge that is transmitted in the sports teaching plays a role in assisting in and supplementing the explicit knowledge. The two knowledge types play a decisive role in sports teaching method. From the current situation of sports teaching, tacit knowledge imposes potential influences on the smooth development of teaching and achievement of teaching goal. The relevant researchers attach great importance to the tacit knowledge, but the research of tacit knowledge in the sports teaching practice is still just a supplement of the current education of sports teaching and the tacit knowledge is not studied as an independent teaching system. This research aims at highlighting the indispensability of tacit knowledge in sports teaching and elaborating its existing way and role, hoping to draw the attention in the reform of sports teaching. 


\section{Meaning and features of tacit knowledge}

(I) Meaning of tacit knowledge

The proposal of tacit knowledge was of cross-time significance in sports research. Famous Physical Chemist and Ideologist Michael Polanyi’s explanation of this concept shaped the complete system of knowledge form. In the late 1950s, Polanyi descriptively explained "tacit knowledge" in his The Study of Man for the first time after many year's painstaking research, at that time, tacit knowledge was translated into “隐性知识”, corresponding to the explicit knowledge. It was also vividly described as “意会知识” and “默会知识” from its expression form. However, people’s understanding of "tacit knowledge" only stayed on the concept, which was regarded as the "book knowledge" that could not be explained in words and not analyzed logically as an important part of knowledge system from the level of epistemology.

Based on this, Polanyi thought the human knowledge was divided into two classes by expression form: systematic knowledge that could be expressed by language, character, picture and mathematical formula and non-systematic knowledge that could not be expressed logically by language, character or symbol and transmitted in a normal form.

(II) Features of tacit knowledge

1. Implicitness of tacit knowledge

The proposal of tacit knowledge didn't mean that tacit knowledge existed in the knowledge independently, but promoted and supplemented the explicit knowledge. Polanyi defined "tacit knowledge" as follows: it is the implicit knowledge in the knowledge system, which only exists in people's hands or minds and can be stood out in case of externalizing behavior, but cannot be expressed by words or formally. Tacit knowledge lacks of intuitive expression form, but widely exists in the society, especially in the industry sector, constantly perfecting the explicit knowledge in a supplementing way. As the hidden knowledge in the thinking, the implicitness of tacit knowledge determines that it is hard to have systematic research.

2. Non-logicality of the implicitness of tacit knowledge 
Tacit knowledge is perceived in an intuitive way and widely exists, but cannot be described by logical language. Horvath defined "tacit knowledge" as follows: what the human know cannot be expressed definitely. For example, in the sports teaching, not all the teaching methods adopted by the teacher are supported by theoretical basis, most of which are just the summary of experience, but can get good teaching effects. However, these experience accumulation is not conducted systematically and a complete system cannot be formed.

\section{Non-criticism of tacit knowledge}

Different from the explicit knowledge, the tacit knowledge has clear logic reasoning process and is critical. It relies on people's intuition or is acquired unconsciously, without rational reflection, so it is not critical with theoretical support. Polanyi listed the tacit knowledge into the research range of "non-critical knowledge", which was the most typical feature of tacit knowledge. Cognition of things is an embodiment of individual's comprehensive factor and the individual's environment and world outlook will affect its cognitive view, which cannot be judged by the standard logical reflection. For example, the sports teacher summarized the teaching method in teaching by virtue of personal experience, which gets significant effects, but cannot be understood by others.

\section{Non-commonality of tacit knowledge}

Tacit knowledge attaches great importance to the individual's cognition of things. Based on the difference of individual perception, tacit knowledge is different in the inner heart of individuals, thus determining the non-commonality of tacit knowledge. For example, mass media conveys information systematically, which is transmitted after extracting and processing. Tacit knowledge cannot be transmitted by this method because the existence of individual difference relies on the perceptual understanding of individual audiences, which is expressed in an indirect way. For example, the tacit knowledge that the sports teacher integrates in the sports teaching is effective for partial students, but hard to understand for partial students. Therefor,e when the teacher conveys knowledge in the form of tacit knowledge, the students with the similar idea can keep up with the teacher's thinking model and better absorb the 
knowledge and the students who cannot understand the tacit knowledge cannot achieve the ideal learning effects.

\section{Existence of tacit knowledge in sports teaching}

(I) Tacit knowledge exists in sports teaching contents

As the sports knowledge conveyed to the students, the sports teaching contents are formed after collection, screening and processing and are the essence of sports knowledge. In sports teaching, many knowledge are explicit, but tacit knowledge penetrates it in the process of knowledge transmission and forms a systematic system after integrating into the theory and existing sports knowledge. For example, "Life lies in movement.” We can understand the importance of sports, but it is a regret for people in the understanding of content that the sports is brought into the range of biotics to form philosophical knowledge. In the sports activity, the content of sports activity can be expressed in place only by integrating the relevant tacit knowledge. With football match as an example, the experienced referee can make an accurate judgment on the changing actions of football players on the football field, which is an embodiment of “intuition” and cannot be explained by explicit knowledge. This means that the sports teacher should continuously enrich their knowledge to expand the sports teaching contents and give play to the tacit knowledge.

(II) Tacit knowledge exists in sports skills

The sports activity has techniques and the sports teaching has procedure. The tacit knowledge existed in the sports skills can be embodied in case of externalizing action, but the starting point of the action mainly relies on the intuition, indicating that the sports skills are intellectual activities with unique nature in the activity process. Specifically, when the tacit knowledge is integrated into the sports skills, the knowledge will be integrated and two knowledge will communicate and exchange with each other in the sports activity, at this time, the sports skills are individualized, which is manifested in the playing of subjective ability. When the individual is in motion, the subjective ability plays a role in guiding the action. The sports body's mastering of sports technique is showed by the embodiment of its tacit knowledge in the sports skills. 


\section{Transmission mode of tacit knowledge in sports teaching}

In the sports teaching, tacit knowledge and explicit knowledge rely on each other, in which the explicit knowledge is clearly expressed by language, character, symbol and other forms and the tacit knowledge cannot be explained in words. From the perspective of pedigree, the two have continuity and can be mutually transformed in the process of information transmission. Considering the long-logical reasoning features of tacit knowledge, its transmission mode is different from that of explicit knowledge. In the classroom teaching of knowledge, the explicit knowledge is more, which is basically the regularity summary of the experiences of predecessors. The specificity of sports education lies in that with the sports skills as the center, in the transmission of knowledge and information, the relevant knowledge is absorbed by the expression form of action and then sublimated in the sports practice by the externalizing form of sports. Therefore, in the sports teaching, the teacher should be tacit with students to form effective interaction of tacit knowledge. In the transmission of tacit knowledge in the sports teaching, the tacit knowledge can be divided into two classes by information transmission effect, that is, "apprenticeship” and "explicit”.

(I) “Apprenticeship” transmission mode of tacit knowledge

Tacit knowledge is mainly used in the teaching level. The sports teacher should realize the existence of tacit knowledge and then change by its habitual transmission mode of explicit knowledge. The sports teacher should master students' absorbing ability of tacit knowledge to adjust its teaching program. In the information transmission process of tacit knowledge, students should absorb and understand the knowledge transmitted by the teacher and convert such knowledge into their knowledge, rather than learn them superficially. Therefore, the sports teacher should rethink constantly in the teaching process and think of the application mode of tacit knowledge in an explicit thinking way so as to improve students' meta-cognitive level. Students should continuously analyze their learning behavior and improve their self-control and self-management ability in the learning process. The tacit knowledge is effective for students to improve their self-control ability. Thus, the “apprenticeship” sports teaching mode produces. 
In the view of Polanyi, “apprenticeship” was one of the most effective ways to transmit tacit knowledge. Some learning skills that cannot be realized by rules should be taught by a method that the master teaches the apprentice, in which there are many tacit understandings, such as non-critical simulation and knowledge transmission based on the cognition of both parties, which are easy for students to effectively absorb the knowledge. In the sports teaching, the use of "apprenticeship" transmission mode of tacit knowledge can get irreplaceable effects that cannot be achieved by explicit transmission mode. Taking martial art teaching as an example, China’s martial arts as an important content in the sports teaching teaches not only martial art skills, but also the martial art culture. Martial art as a part of Chinese traditional culture pursues the cultural spirit of martial art and these cultural deposits cannot be expressed only by martial art movements. Therefore, in the martial art teaching, the teacher can use multimedia to create the martial art situation to make students simulate the teachers' movement in the music full of artistic conception and feel the cultural connotation and charm of martial art. "Apprentice” is originally a "tutorial” teaching mode that a teacher guides a few students. When it is used in the sports teaching, the teacher "teaches students in deeds" to make the teaching mode flexible and diversified, thus playing a role of subtly guiding students.

(I) "Explicit” transmission mode of tacit knowledge

In the personal knowledge system of sports teacher, the tacit knowledge is an important part and is often explicated by sports skills. However, in many practical teachings of sports, the sports teacher is mere the expression of skills and cannot hold the language expression. At this time, the use of tacit knowledge can property convey the teaching content to students and make them understand tacitly. The experienced sports teacher often takes the things that the students are familiar with as a metaphor to properly express the teaching content and make students understand the knowledge more intuitively. For example, when students are learning Fosbury Flop, the important problem is that the hip joints are hard to open, so students often touch the bar by hip. To make students act accurately and in place, in addition to do posture, the teacher should use metaphor to make students understand the actions of Fosbury Flop and 
then correct their action by perception. When students do a wrong action, the sports teacher metaphorizes the action as a "shoe-shaped gold ingot", thus students can understand their crucial reason quickly and accept it easily. Using this explicit transmission mode of tacit knowledge, the sports teacher should rethink constantly and make adjustment and modification according to the specific teaching situation to get good teaching effects.

\section{Conclusion:}

In conclusion, from the perspective of discipline teaching, the reform of sports teaching is not just to change in form, but to change the teaching method and means from the teaching concept. As a part of sports teaching, the important role of tacit knowledge in sports teaching is more and more concerned. The tacit knowledge exists in the teaching of sports principle and sports skills and plays an important role in conveying the information.

\section{References:}

[1] Xiong Yanfei. Discussion on Tacit Knowledge in Sports Teaching and its Transmission Mode[J]. Journal of Hebei Institute of Physical Education, 2006 (12). [2]Harry Collins. Tacit and explicit knowledge [M]. Chicago: The University of Chicago Press, 2010 (04).

[3] Miao Qing. Discussion on Tacit Knowledge of Sports Teacher under the Concept of New Course Standards[J]. The Science Education Article Collects, 2007 (08).

[4] Robert Scott Kretchmar. Practical philosophy of sport [M]. Champaign, IL: Human Kinetics, 1994.

[5] Shi Jiangnian, Zhang Jianhua, Zhang Huizhen. On Existence and Transmission of Tacit Knowledge in Sports Teaching [J]. Journal of Physical Education, 2014.21 (01). 